\title{
A NOTE ON RECURSIVE ESTIMATOR OF THE DENSITY FUNCTION WHICH IS NOT NECESSARY CONTINUOUS
}

\author{
M. M. EL-FAHHAM
}

Summary. Consider a sequence $X_{1}, X_{2}, \ldots, X_{n}$ of independent, identically distributed random variables with nukown density function $f$, which with its first derivative are not necessarily continuous, and let

$$
f_{n}^{\star}(x)=\left(n^{2} h_{n}\right)^{-1 / 2} \sum_{j=1}^{n} h_{j}^{-1 / 2} K\left(\frac{x-X_{j}}{h_{j}}\right)
$$

be the recussive kemel estimator of $f$. It will be shown, under certain additional regularity conditions on $K$ and $|h(n)|$, that, $M I S E\left[f_{n}^{\star}(x)\right]=0\left(n^{-1 / 5}\right)$ if $f$ and $f^{\prime}$ are continuous, where as $M I S E\left[f_{n_{1}}^{\star}(x)\right]=0\left(n^{-3 / 4}\right)$ if $f$ is continuous and $f^{\prime}$ is not and MISE $\left[f_{n_{2}}^{\star}(x)\right]=0\left(n^{-1 / 2}\right)$ if $f$ and $f^{\prime}$ are not continuous.

\section{Introduction:}

Consider a sequence $X_{1}, X_{2}, \ldots, X_{n}$ of $n$ independent, identically distributed random variables (i.i.d.) with unknown density function (d.f.) $f(x)$. A class of density estimators, known as Kernel estimators, which has been widely studied by Parzen [1] and Rosenblatt [2] may be defined as follows:

$$
\hat{f}_{n}(x)=(n h)^{-1} \sum_{j=1}^{n} K\left(\frac{\not 2-X_{j}}{h}\right),
$$

Parzen has shown that if the Kernel function $K($.) satisfies certain conditions, including $\int_{-\infty}^{\infty} K(y) d y=1$ and $h=h(n)$ is a bounded and nonincreasing function of $n$ such that $h(n)=0\left(n^{-1}\right)$, then $\hat{f}(2)$ is a mean squared error consistnet estimator of $f(x)$ at all continuity points $x$ of $f$.

E. J. Wegman and II. I. Davies [3] considered a modified estimator of the form

$$
f_{n}^{*}(x)=\left(n^{2} h_{n}\right)^{-1 / 2} \sum_{j=1}^{n}\left(h_{j}\right)^{-1 / 2} K\left(\frac{x-X_{j}}{h_{j}}\right) .
$$

Received April 9, 1990 
They established laws of the iterated logarithm for density estimators as well as the asymptotic distribution results using the almost sure invariance principle.

In this article we shall discuss classes of estimators of the form $f_{n}^{*}(x)$ for an unknown d.f. $f(x)$ which with its first derivative are not necessarily continuous. As a measure of improvement, many authors use the mean integrated square error given by

$$
\operatorname{MISE}\left[f_{n}^{*}(x)\right]=E \int-\infty^{\infty}\left[f_{n}^{*}(x)-f(x)\right]^{2} d x
$$

Let

$$
M_{1} I S E\left[f_{n}^{*}(x)\right]=E \int_{-\infty}^{\infty}\left[f_{n}^{*}(x)-B(f(x))\right]^{2} d x
$$

where

$$
B(f(x))=E\left[f_{n}^{*}(x)\right]-f(x)
$$

Hence

$$
M I S E\left[f_{n}^{*}(x)\right]=M_{1} I S E\left[f_{n}^{*}(x)\right]+(1-B)^{2}\|f(x)\|^{2} .
$$

Consider a recursive Kernel estimator of the form (1) of $f$ based on the random sample. Assuming $K($.$) is a some suitable Kernel function satisfying the following con-$ ditions:

$$
\text { (A) } \begin{cases}\sup _{x}|K(x)|<\infty, & \lim _{x \rightarrow \pm \infty}|x K(x)|=0, \\ \int_{-\infty}^{\infty}|K(x)| d x<\infty \text { and } & \int_{-\infty}^{\infty} x^{2} K(x) d x<\infty,\end{cases}
$$

and $h(n)$ is a sequence of nonincreasing positive constants converging to zero as $n$ tends to $\infty$.

As symbols, we shall use $f_{n_{0}}^{*}(x)$ as an estimator for $f$ when $f$ and $f^{\prime}$ are continuous, if $f$ is continuous but $f^{\prime}$ is not, then we take $f_{n_{1}}^{*}(x)$ as an estimator for $f$ and $f_{n_{2}}^{*}(x)$ as an estimator for $f$ when $f$ and $f^{\prime}$ are not continuous.

Main results:

Theorem 1. Assum that $K($.$) is a Borel function satisfies conditions (A)$ and $n h_{n} \rightarrow \infty$ as $n \rightarrow \infty$, if $f(x), f^{\prime}(x)$ are continous and $f^{\prime \prime}$ is square integrable, then

$$
\begin{aligned}
& \operatorname{MISE}\left[f_{n_{0}}^{*}(x)\right]=C_{1}\left\{\left(n h_{n}\right)^{-1}\|K(t)\|^{2}+0\left(\left(n h_{n}\right)^{-1}\right)+\left[1-\left(n^{2} h_{n}\right)^{-1 / 2} r_{n}\right]^{2}\right. \\
& \left.\times\|f(2)\|^{2}+\frac{1}{2}\left(n h_{n}\right)^{-1}\left[\sum_{j=1}^{n} h_{j}^{5}\left\|t K^{1 / 2}(t)\right\|^{4}\left\|f^{\prime \prime}(x)\right\|^{2}+0\left(h_{j}^{5}\right)\right]\right\} ;
\end{aligned}
$$

where $0<c_{1}<1,\|g(t)\|^{2}=\int g^{2}(t) d t$ and

$$
r_{n}=\sum_{j=1}^{n} h_{j}^{1 / 2}
$$

If $\left(n^{2} h_{n}\right)^{-1 / 2} r_{n} \rightarrow B<\infty$ and $\left(n h_{n}\right)^{-1} \sum_{j=1}^{n} h_{j}^{5} \rightarrow 0$ as $n \rightarrow \infty$, 
then

and hence

$$
\operatorname{MISE}\left[f_{n}^{*}(x)\right] \rightarrow(1-B)^{2}\|f(x)\|^{2} \text { as } n \rightarrow \infty
$$

$$
M_{1} I S E\left[f_{n}^{*}(x)\right] \rightarrow 0 \text { as } n \rightarrow \infty \text {. }
$$

In particular if $h_{j}=0\left(n^{-r}\right), j=1,2, \ldots, n ; r>0$, then

$$
M_{1} I S E\left[f_{n}^{*}(x)\right]=0\left(n^{r-1}\right)+0\left(n^{-4 r}\right)
$$

and hence the asymplotic optimum value $r_{0}$ of $r$ is $\frac{1}{5}$ and then we get minimum $M_{1} I S E\left[f_{n_{0}}^{*}(x)\right]=0\left(n^{-4 / 5}\right)$.

Theorem 2. Suppose $K($.$) and h_{n}$ satisfy conditions of theorem 1.

If $f(x)$ is continuous, $f^{\prime}$ has finite points of discontinuity $\left(b_{1}, b_{2}, \ldots, b_{\ell}\right)$ and $f^{\prime \prime}$ is square integrable, then

$$
\begin{aligned}
M_{1} I S E\left[f_{n_{1}}^{*}(x)\right]=C_{2}\left\{M_{1} I S E\left[f_{n_{0}}^{*}(x)\right]+\frac{4 \Delta}{n}\left(\sum_{j=1}^{n} h_{j}^{3}\right) \int_{-\infty}^{0}\left[\int_{-\infty}^{y}(y-t) K(t) d t\right]^{2} d y\right. \\
\left.+O\left(\frac{1}{n} \sum_{j=1}^{n} h_{h_{j}}^{3}\right)\right\}
\end{aligned}
$$

where $0<c_{2}<1, \Delta=\sum_{j=1}^{\ell} \Delta_{j}^{2}$,

$$
\Delta_{j}=f^{\prime}\left(b_{j}^{-}\right)-f^{\prime}\left(b_{j}^{+}\right), j=1,2, \ldots, n,
$$

If $\frac{1}{n} \sum_{j=1}^{n} h_{j}^{3} \rightarrow 0$ as $n \rightarrow \infty$, then as $n \rightarrow \infty$, the estimation $f_{n_{1}}^{*}(x)$ is consistent in $M_{1} I S E$ sense, specially if $h_{j}=0\left(n^{-r}\right), j=1,2, \ldots, n, r>0$, then $M_{1} I S E\left[f_{n_{1}}^{*}(x)\right]=$ $0\left(n^{r-1}\right)+O\left(n^{-3 r}\right)$ and we get the asymptotic optimum value $r_{0}$ of $r$ is $\frac{1}{4}$, and hence minimum $M_{1} I S E\left[f_{n_{1}}^{*}(x)\right]=0\left(n^{-3 / 4}\right)$.

Theorem 3. Let $K($.$) and h_{n}$ satisfy conditions of theorem 1.

If $f$ has $k(k \geq 0)$ points of discontinuity, $-\infty=a_{0}<a_{1}<a_{2}<\ldots<a_{k}<a_{k+1}=$ $\infty$, and for cach $i=1, \ldots, k+1, f^{\prime}$ has $\ell_{i}\left(\ell_{i} \geq 0\right)$ points of discontinuity.

$$
\begin{gathered}
a_{i-1}=b_{i_{0}}<b_{i_{1}}<b_{i_{2}}<\ldots<b_{i_{\ell_{i}}}<b_{i_{\ell_{i+1}}}=a_{i} \text {, also } \\
\int_{-\infty}^{\infty}\left|f^{\prime}(x)\right| d x<\infty, \quad \int_{-\infty}^{\infty}\left|f^{\prime \prime}(x)\right|^{2} d x<\infty
\end{gathered}
$$

then

$$
\begin{aligned}
& M_{1} \operatorname{ISE}\left[\int_{n}^{*}(x)\right]<c_{2} M_{1} \operatorname{ISE}\left[\int_{n_{1}}^{*}(x)\right]+\frac{4 \delta}{n}\left(\sum_{j=1}^{n} h_{j}\right) \int_{0}^{\infty}\left|\int_{y}^{\infty} K(x) d x\right|^{2} d y \\
& \quad+0\left(\frac{1}{n} \sum_{j=1}^{n} h_{j}\right)
\end{aligned}
$$


where $\delta=\sum_{i=1}^{k} \delta_{i}^{2}, \quad \delta_{i}=f\left(a_{i}^{-}\right)-f\left(a_{i}^{+}\right)$,

$$
\begin{aligned}
\Delta & =\sum_{i=1}^{k+1} \sum_{j=1}^{\ell_{i}+1} \Delta_{i j}^{2} & \text { and } & \triangle_{i j}=f^{\prime}\left(b_{i j}^{-}\right)-f^{\prime}\left(b_{i j}^{+}\right), \\
i & =1, \cdots, k+1 & \text { and } & j=1, \cdots, \ell_{i}+1 .
\end{aligned}
$$

If $\frac{1}{n} \sum_{j=1}^{n} h_{j} \rightarrow 0$ as $n \rightarrow \infty$, then the estimation $f_{n_{2}}^{*}(x)$ is consistent in $M_{1} I S E$ sense. In particular if $h_{j}=0\left(n^{-r}\right), j=1,2, \cdots, n, r>0$, then $M_{1} I S E\left[f_{n_{2}}^{*}(x)\right]=0\left(n^{r-1}\right)+0\left(n^{-r}\right)$ and also we get ro $=\frac{1}{2}$ and minimum $M_{1} I S E\left[f_{n_{2}}^{*}(x)\right]=0\left(n^{-1 / 2}\right)$.

Proof: It is sufficient to prove theorem 3 which implies theorem 1 and 2.

We can see that

$$
\begin{aligned}
\operatorname{MISE}\left[f_{n_{2}}^{*}(x)\right]= & E \int_{-\infty}^{\infty}\left[f_{n}^{*}(x)-f(x)\right]^{2} d x \\
= & E \int_{-\infty}^{\infty}\left[\left(n^{2} h_{n}\right)^{-1 / 2} \sum_{j=1}^{n} h_{j}^{-1 / 2} K\left(\frac{x-X_{j}}{h_{j}}\right)-f(x)\right]^{2} d x \\
= & \int_{-\infty}^{\infty}\left[\left(h^{2} h_{n}\right)^{-1} \sum_{j=1}^{n} h_{j}^{-1} E K^{2}\left(\frac{x-X_{j}}{h_{j}}\right)\right. \\
& +2\left(n^{2} h_{n}\right)^{-1} \sum_{1=i<j}^{n}\left(h_{i} h_{j}\right)^{-1 / 2} E K\left(\frac{x-X_{i}}{h_{i}}\right) K\left(\frac{x-X_{j}}{h_{j}}\right) \\
& \left.-2 f(x)\left(n^{2} h_{n}\right)^{-1 / 2} \sum_{j=1}^{n}\left(h_{j}\right)^{-1 / 2} E K\left(\frac{x-X_{j}}{h_{j}}\right)+f^{2}(x)\right] d x .
\end{aligned}
$$

First

$$
\begin{aligned}
\int_{-\infty}^{\infty} h_{j}^{-1} E K^{2}\left(\frac{2-X_{j}}{h_{j}}\right) d x & =\int_{-\infty}^{\infty} \int_{-\infty}^{\infty} h_{j}^{-1} K^{2}\left(\frac{x-y}{h_{j}}\right) f(y) d y d x \\
& =\int_{-\infty}^{\infty} \int_{-\infty}^{\infty} K^{2}(t) f\left(x-t h_{j}\right) d t d x \\
& =\int_{-\infty}^{\infty} K^{2}(t) d t+0(1) .
\end{aligned}
$$

Second

$$
\int_{-\infty}^{\infty} h_{j}^{-1} f(x) E K\left(\frac{x-X_{j}}{h_{j}}\right) d x=\int_{-\infty}^{\infty} f(x) \int_{-\infty}^{\infty} K(t) f\left(x-t h_{j}\right) d t d x
$$


At last

$$
\begin{aligned}
& \sum_{1=i<j}^{n} \int_{-\infty}^{\infty}\left(h_{i} h_{j}\right)^{-1 / 2} E K\left(\frac{x-X_{i}}{h_{i}}\right) E K\left(\frac{x-X_{j}}{h_{j}}\right) d x \\
= & \sum_{1=i<j}^{n}\left(h_{i} h_{j}\right)^{1 / 2}\left\{\int_{-\infty}^{\infty} \int_{-\infty}^{\infty} \int_{-\infty}^{\infty} K(t) K(s)\left[f\left(x-t h_{i}\right)-f(x)\right]\right. \\
& \times\left[f\left(x-s h_{j}\right)-f(x)\right] d t d s d x+\int_{-\infty}^{\infty} \int_{-\infty}^{\infty} \int_{-\infty}^{\infty} K(t) K(s) f \cdot(x)\left[f\left(x-t h_{i}\right)\right. \\
& \left.\left.+f\left(x-s h_{j}\right)\right] d t d s d x-\int_{-\infty}^{\infty} f^{2}(x) d x\right\} .
\end{aligned}
$$

It is easy to see that the second term in the last equation is equal to

$$
r_{n} \sum_{j=1}^{n} h_{j}^{1 / 2} \int_{-\infty}^{\infty} f(x) \int_{-\infty}^{\infty} K(t) f\left(x-t h_{j}\right) d t d x ; r_{j}=\sum_{j=1}^{n} h_{j}^{1 / 2} .
$$

from (3), (4), (5) and (6) we get

$$
\begin{aligned}
M I S E & \left.f_{n_{2}}^{w_{2}}(x)\right]=\left(n h_{n}\right)^{-1} \int_{-\infty}^{\infty} K^{2}(t) d t \\
& +2\left(n^{2} h_{n}\right)^{-1} \sum_{1=i<j}^{n}\left(h_{i} h_{j}\right)^{1 / 2} \int_{-\infty}^{\infty} J\left(x, h_{i}\right) J\left(x, h_{j}\right) d x \\
& +2 r_{n}\left(n^{2} h_{n}\right)^{-1} \sum_{j=1}^{n} h_{j}^{1 / 2} \int_{-\infty}^{\infty} \int_{-\infty}^{\infty} K(t) f(x) f\left(x-t h_{j}\right) d t d x \\
& -\left(n^{2} h_{n}\right)^{-1} r_{n}^{2} \int_{-\infty}^{\infty} f^{2}(x) d x-2\left(n^{2} h_{n}\right)^{-1 / 2} \sum_{j=1}^{n} h_{j}^{1 / 2} \int_{-\infty}^{\infty} f(x) J\left(x, h_{j}\right) d x \\
& -2\left(n^{2} h_{n}\right)^{-1 / 2} r_{n} \int_{-\infty}^{\infty} f^{2}(x) d x+\int_{-\infty}^{\infty} f^{2}(x) d x+0\left(\left(n h_{n}\right)^{-1}\right),
\end{aligned}
$$

where $J(x, h)=\int_{-\infty}^{\infty} K(t)[f(x-t h)-f(x)] d t$. Then

$$
\begin{aligned}
M I S E\left[f_{n_{2}}^{*}(x)\right]= & \left(n h_{n}\right)^{-1} \int_{-\infty}^{\infty} \mathbb{K}^{2}(t) d l \\
& +2\left(n^{2} h_{n}\right)^{-1} \sum_{1=i<j}^{n}\left(h_{i} h_{j}\right)^{-1 / 2} \int_{-\infty}^{\infty} J\left(x, h_{i}\right) J\left(x, h_{j}\right) d x \\
& -2\left(n^{2} h_{n}\right)^{-1 / 2}\left[1-r_{n}\left(n^{2} h_{n}\right)^{-1 / 2}\right] \sum_{j=1}^{n} h_{j}^{1 / 2} \int_{-\infty}^{\infty} f(x) J\left(x, h_{j}\right) d x \\
& +\left[1-\left(n^{2} h_{n}\right)^{-1 / 2} r_{n}\right]^{2} \int_{-\infty}^{\infty} f^{2}(x) d x+0\left(\left(n h_{n}\right)^{-1}\right) .
\end{aligned}
$$


By using Hölder's inequality we get

$$
\begin{aligned}
& \operatorname{MISE}\left[f_{n_{2}}(x)\right] \leq\left(n h_{n}\right)^{-1}\|K(t)\|^{2}+\left[1-\left(n^{2} h_{n}\right)^{-1 / 2} r_{r_{n}}\right]^{2}\|f(x)\|^{2} \\
& +2\left(n^{2} h_{n}\right)^{-1} \sum_{1=i<j}^{n}\left(h_{i} h_{j}\right)^{+1 / 2}\left\|J\left(x, h_{i}\right)\right\|\left\|J\left(x, h_{j}\right)\right\|+0\left(\left(n h_{n}\right)^{-1}\right) .
\end{aligned}
$$

By using Taylor series-like expansions for $f$ satisfying conditions of our theorem

a) For $b_{i j_{1}-1}<x<b_{i j_{1}}, b_{i j_{2}-1}<x-t h<b_{i j_{2}}, 1 \leq j_{1} \leq l_{i}+1,1 \leq j_{2} \leq l_{i}+1$, $i=1,2, \cdots, k+1$

$$
\begin{gathered}
f(x-t h)-f(x)+t h f^{\prime}(x)-h^{2} \int_{0}^{t}(t-s) f^{\prime \prime}(x-s h) d s \\
=\left\{\begin{array}{lc}
0 & j_{1}=j_{2} \\
\sum_{r=j_{2}}^{j_{2}-1}\left(b_{i r}-x+t h\right) \Delta_{i r}, & j_{1}<j_{2} \\
\sum_{r=j_{2}-1}^{j_{1}-1}\left(b_{i r}-x+t h\right) \Delta_{i r}, & j_{1}>j_{2}
\end{array}\right.
\end{gathered}
$$

b) For $b_{i_{1} j_{2}-1}<2<b_{i_{1} j_{1}}, b_{i_{2} j_{2}-1}<2-t h<b_{i_{2} j_{2}}, 1 \leq j_{1} \leq 1_{i_{1}}+1,1 \leq j_{2} \leq 1_{i_{2}}+1$,

$1 \leq i_{1} \leq k+1,1 \leq i_{2} \leq k+1, i_{1} \neq i_{2}$

$$
\begin{aligned}
& f(x-t h)-f(x)+t h f^{\prime}(x)-h^{2} \int_{0}^{l}(t-s) f^{\prime \prime}(x-s h) d s \\
& =\left\{\begin{array}{c}
\text { (i) } \quad \sum_{r=j_{1}}^{l_{i_{1}}+1}\left(b_{i_{2} r}-x+t h\right) \Delta_{i_{1} r}+\sum_{r=i_{1}+1}^{i_{2}-1} \sum_{r^{\prime}=1}^{l_{l}+1}\left(b_{r r^{\prime}}-x+t h\right) \Delta_{r r}, \\
\text { (ii) }+\sum_{r=j_{2}}^{j_{3}-1}\left(b_{i_{2} r}-x-t h\right) \Delta_{i_{2} r}-\sum_{\mu=i_{2}}^{i_{2}-1} \delta_{\mu} \text { if } i_{1}<i_{2} \\
\text { (iii) }-\sum_{r=j_{2}}^{l_{i_{2}+1}+1}\left(b_{i_{2} r}-x+t h\right) \Delta_{i_{2} r}-\sum_{r=i_{2}+1}^{i_{1}-1} \sum_{r^{\prime}=1}^{l_{l}+1}\left(b_{r r^{\prime}}-x+t h\right) \Delta_{r r} \\
-\sum_{r=1}^{j_{1}-1}\left(b_{i_{2} r}-x+t h\right) \Delta_{i_{1} r}+\sum_{\mu=i_{2}}^{i_{1}-1} \delta_{\mu} \text { if } i_{1}>i_{2}
\end{array}\right.
\end{aligned}
$$

Let

$$
G(x, h)=h^{2} \int_{-\infty}^{\infty} K(t) \int_{0}^{t}(t-s) f^{\prime \prime}(x-s h) d s d t,-\infty<x<\infty
$$




$$
\begin{aligned}
H_{i j 1}(x, h)= & \sum_{r=j}^{l_{i}+1} \Delta_{i r} \int_{-\infty}^{\frac{x-b_{i r}}{h}}\left(b_{i r}-x+t h\right) K(t) d t \\
& +\sum_{r=i+1}^{k+1} \sum_{r^{\prime}=1}^{l_{\ell+1}} \Delta_{r r^{\prime}} \int_{-\infty}^{\frac{x-b_{r r^{\prime}}}{h}}\left(b_{r r^{\prime}}-x+t h\right) K(t) d t \\
H_{i j 2}(x, h)= & -\sum_{r=1}^{j-1} \Delta_{i r} \int_{\frac{s-b_{i x}}{h}}^{\infty}\left(b_{i r}-x+t h\right) K(t) d t \\
& -\sum_{r=1}^{i-1} \sum_{r^{\prime}=1}^{l_{l+1}} \Delta_{r r^{\prime}} \int_{\frac{x-b_{r} x^{\prime}}{h}}^{\infty}\left(b_{r r^{\prime}}-x+t h\right) K(t), \\
b_{i j-1}<x<b_{i j} ; j=1,2, \cdots, l_{i+1}, i=1,2, \cdots, k+1, & \sum_{i=1} \delta_{\mu}\left\{1-W\left(\frac{x-a_{\mu}}{h}\right)\right\}, a_{i-1}<x<a_{i}, i=1, \cdots, k+1, \\
V_{i 1}(x, h)= & \sum_{i=1}^{k} \delta_{\mu=i} \delta_{\mu} W\left(\frac{x-a_{\mu}}{h}\right), a_{i-1}<x<a_{i}, i=1, \cdots, k .
\end{aligned}
$$

Where $W(y)=\int_{-\infty}^{y} \mathbb{K}(t) d t,-\infty<y<\infty$

Further let, for $j=1, \cdots, l_{i}+1, i=1, \cdots, k+1$

$$
\begin{aligned}
H_{i j}(x, h) & =H_{i j 1}(x, h)+H_{i j 2}(x, h) \text { and } \\
V_{i}(x, h) & =V_{i 1}(x, h)+V_{i 2}(x, h) .
\end{aligned}
$$

\section{Lemma 1.}

$$
\lim _{h \rightarrow 0} \frac{1}{h^{4}} \int_{-\infty}^{\infty} G(x, h) d x=\frac{1}{4}\left(\int_{-\infty}^{\infty} t^{2} K(t) d t\right)^{2} \cdot \int_{-\infty}^{\infty}\left(f^{\prime \prime}(x)^{2} d x\right.
$$

\section{Lemmmen 2.}

$$
\begin{gathered}
\lim _{h \rightarrow 0} \frac{1}{h^{3}} \int_{b_{i j-1}}^{b_{i j}} H_{i j}^{2}(x, h) d x=\left(\triangle_{i j}^{2}+\Delta_{i j-1}^{2}\right) \int_{-\infty}^{0}\left\{\int_{-\infty}^{y}(y-t) K(t) d t\right\}^{2} d y \\
1 \leq j \leq 1_{i+1}, i=1, \cdots, k+1 .
\end{gathered}
$$

Lemma 3.

$$
\lim _{h \rightarrow 0} \frac{1}{h} \int_{a_{i-1}}^{a_{i}} V_{i}^{2}(x, h) d x=\left(\delta_{i}^{2}+\delta_{i-1}^{2}\right) \int_{0}^{\infty}(1-W(y))^{2} d y, i=1, \cdots, k+1 .
$$

Lemma 4.

$$
\begin{aligned}
& \lim _{h \rightarrow 0} \frac{1}{h^{3}} \int_{b_{i j-1}}^{b_{i j}} G(x, h) H_{i j}(x, h) d x=\lim _{h \rightarrow 0} \frac{1}{h} \int_{a_{i-1}}^{a_{i}} G(x, h) V_{i}(x, h) d x \\
= & \lim _{h \rightarrow 0} \frac{1}{h} \int_{b_{i j-1}}^{b_{i j}} H_{i j}(x, h) V_{i}(x, h) d x=0,1 \leq j \leq 1_{i=1}, i=1, \cdots, k+1
\end{aligned}
$$


Proofs of these lemmas given by Eden, C.V. [4].

We can see that

$$
\begin{aligned}
& \int_{-\infty}^{\infty} J^{2}(x, h) d x \\
& =\int_{-\infty}^{\infty}\left\{\int_{-\infty}^{\infty} K(t)[f(x-t h)-f(x)] d t\right\}^{2} d x \\
& =\sum_{i=1}^{k+1} \sum_{j=1}^{\ell_{i+1}} \int_{b_{i j-1}}^{b_{i j}}\left\{G(x, h)+H_{i j}(x, h)+V_{i}(x, h)\right\}^{2} d x \\
& =\sum_{i=1}^{k+1} \sum_{j=1}^{\ell_{i+1}} \int_{b_{i j-1}}^{b_{i j}} G^{2}(x, h) d x+\sum_{i=1}^{k+1} \sum_{j=1}^{\ell_{i+1}} \int_{b_{i j-1}}^{b_{i j}} H_{i j}^{2}(x, h) d x \\
& +\sum_{i=1}^{l+1} \sum_{j=1}^{\ell_{i+1}} \int_{b_{i j-1}}^{b_{i j}} V_{i}^{2}(x, h) d x+2 \sum_{i=1}^{l+1} \sum_{j=1}^{\ell_{i+1}} \int_{b_{i j-1}}^{b_{i j}} G(x, h) H_{i j}(x, h) d x \\
& +2 \sum_{i=1}^{k+1} \sum_{j=1}^{\ell_{i+1}} \int_{b_{i j-1}}^{b_{i j}} G(x, h) V_{i}(x, h) d x+2 \sum_{i=1}^{k+1} \sum_{j=1}^{\ell_{i+1}} \int_{b_{i j-1}}^{b_{i j}} H_{i j}(x, h) V_{i}(x, h) d x \\
& =\int_{-\infty}^{\infty} G^{2}(x, h) d x+\sum_{i=1}^{k+1} \sum_{j=1}^{\ell_{i+1}} \int_{b_{i j-1}}^{b_{i j}} H_{i j}^{2}(x, h) d x \\
& +\sum_{i=1}^{l+1} \int_{a_{i-1}}^{a_{i}} V_{i}^{2}(x, h) d x+2 \sum_{i=1}^{k+1} \sum_{i=1}^{\ell_{i+1}} \int_{b_{i j-1}}^{b_{i j}} G(x, h) H_{i j}(x, h) d x \\
& +2 \sum_{i=1}^{k+1} \int_{a_{i-1}}^{a_{i}} G(x, h) V_{i}(x, h) d x+2 \sum_{i=1}^{k+1} \sum_{j=1}^{\ell_{i+1}} \int_{b_{i j-1}}^{b_{i j}} H_{i j}(x, h) V_{i}(x, h) d x \text {. }
\end{aligned}
$$

Then from lemmas (1),(2),(3) and (4), we get

$$
\begin{aligned}
\int_{-\infty}^{\infty} J^{2}(x, h) d x= & \frac{h^{4}}{4}\left(\int_{-\infty}^{\infty} t^{2} K(t) d t\right)^{2} \int_{-\infty}^{\infty}\left(f^{\prime \prime}(x)\right)^{2} d x+0\left(h^{4}\right) \\
& +h^{3} \sum_{i=1}^{k+1} \sum_{j=1}^{l_{i}+1}\left(\triangle_{i j}^{2}+\triangle_{i j-1}^{2}\right) \int_{-\infty}^{0}\left\{\int_{-\infty}^{y}(y-t) K(t) d t\right\}^{2} d y \\
& +0\left(h^{3}\right)+h \sum_{i=1}^{k+1}\left(\delta_{i}^{2}+\delta_{i-1}^{2}\right) \int_{0}^{\infty}(1-W(y))^{2} d y+0(h) .
\end{aligned}
$$

But from the fact that

$$
\sum_{i=1}^{k+1} \sum_{j=1}^{l_{i}+1} \triangle_{i j-1}^{2}=\sum_{i=1}^{k+1} \sum_{j=1}^{l_{i}+1} \triangle_{i j}^{2}=\Delta,
$$


and

$$
\sum_{i=1}^{k+1} \delta_{i-1}^{2}=\sum_{i=1}^{k+1} \delta_{i}^{2}=\delta,
$$

We have

$$
\begin{aligned}
\int_{-\infty}^{\infty} J^{2}\left(x, h_{i}\right) d x= & \frac{1}{4} h_{j}^{4}\left\|t K^{1 / 2}(t)\right\|^{4} \cdot\left\|f^{\prime \prime}(x)\right\|^{2} \\
& +0\left(h_{j}^{4}\right)+2 h_{j}^{3} \Delta \int_{-\infty}^{0}\left(\int_{-\infty}^{y}(y-t) K(t) d t\right)^{2} d y+0\left(h_{j}^{3}\right) \\
& +2 h_{j} \delta \int_{0}^{\infty}(1-W(y))^{2} d y+0\left(h_{j}\right) .
\end{aligned}
$$

From (7) and (8) we get

$$
\begin{aligned}
\operatorname{MISE}\left[f_{n_{2}}^{*}(x)\right] \leq & \left(n h_{n}\right)^{-1}\|K(t)\|^{2}+0\left(n h_{n}\right)^{-1}+\left[1-\left(n^{2} h_{n}\right)^{-1 / 2} r_{n}\right]^{2}\|f(x)\|^{2} \\
& +2\left(n h_{n}\right)^{-1}\left[\frac{1}{4}\left\|t K^{1 / 2}(t)\right\|^{4} \cdot\left\|f^{\prime \prime}\right\|^{2}\left(\sum_{j=1}^{n} h_{j}^{5}\right)+0\left(h_{j}^{5}\right)\right. \\
& +2 \Delta\left(\sum_{j=1}^{n} h_{j}^{4}\right) \int_{-\infty}^{0}\left\{\int_{-\infty}^{y}(y-t) K(t) d t\right\}^{2} d y \\
& +0\left(h_{j}^{4}\right)+2 \delta \int_{0}^{\infty}\{1-W(y)\}^{2} d y\left(\sum_{j=1}^{n} h_{j}^{2}\right)+0\left(h_{j}^{2}\right) .
\end{aligned}
$$

Which completes the proof of the theorem.

\section{References}

[1] Parzen, E., "On estimation of a probability density and mode". Ann. Math. Statist. 33, 1065-1076, 1962.

[2] Rosenblatt, M., "The central limit theorem for mixing sequences of random variables". Z. Wahrscheinl, chkeitsch., $12,155-171,1956$.

[3] Wegman, E. J. and J. I. Davies. "Remarks on some recursive estimators of a probability density". Ann. Math. Statist., Vol. 7, 316327, 1979.

[4] Van Eeden C., "Mean integrated squared error of Kernel estimators when the density and its derivative are not necessarily continuous". Roppart de Recherches no. 82, Département de mathematiques et de Statistique, Université de Montréal. 1984.

Dept. of Mathematics, Faculty of Science, Cairo University, Cairo, Egypt. 